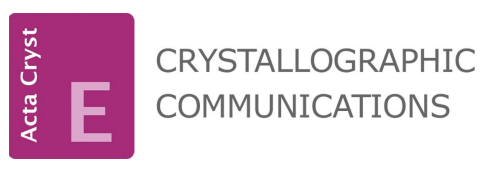

ISSN 2056-9890

Received 10 August 2016

Accepted 29 August 2016

Edited by G. Smith, Queensland University of Technology, Australia

Keywords: crystal structure; aryl cyclohexyl nonanoid; M. malabarica; antiproliferative activity; atom disorder and refinement; hydrogen bonding.

CCDC reference: 1501296

Supporting information: this article has supporting information at journals.iucr.org/e

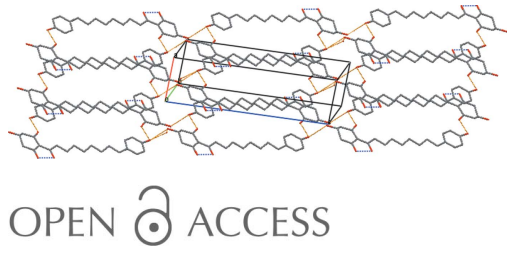

\section{Crystal structure of an aryl cyclohexyl nonanoid, an antiproliferative molecule isolated from the spice Myristica malabarica}

\author{
Ajoy Kumar Bauri, ${ }^{a}$ Sabine Foro ${ }^{\mathrm{b}}$ and Nhu Quynh Nguyen Do ${ }^{\mathrm{c} *}$ \\ ${ }^{\mathbf{a} B i o-O r g a n i c ~ D i v i s i o n, ~ B h a b h a ~ A t o m i c ~ R e s e a r c h ~ C e n t r e, ~ T r o m b a y, ~ M u m b a i ~ 400085, ~ I n d i a, ~}{ }^{\mathbf{b}}$ Institute of Materials \\ Science, Darmstadt University of Technology, Alarich-Weiss-Strasse 2, D-64287 Darmstadt, Germany, and ${ }^{\mathbf{c}}$ Accident and \\ Emergency Department, Franco Vietnamese Hospital, 7 Nguyen Luong Bang Street, Ho Chi Minh City, Vietnam. \\ *Correspondence e-mail: nguyendonhuquynh@yahoo.com
}

The title compound, $\mathrm{C}_{21} \mathrm{H}_{26} \mathrm{O}_{5}$, an aryl cyclohexyl nonanoid \{systematic name: 3,5-dihydroxy-2-[9-(4-hydroxyphenyl)nonanoyl]cyclohexa-2,4-dien-1-one], extracted from the spice plant Myristica malabarica comprises two ring components, a 4-hydroxyphenyl moiety and a 3,5-dihydroxycyclohexa-2,4dienone moiety linked by a nonanoyl chain. The molecule has an extended essentially planar conformation stabilized by an intramolecular hydroxy $\mathrm{O}-$ $\mathrm{H} \cdots \mathrm{O}_{\text {carbonyl }}$ hydrogen bond, giving a dihedral angle between the two ring systems of $6.37(15)^{\circ}$. The $\mathrm{C}, \mathrm{O}$ and $\mathrm{H}$ atoms associated with one of the hydroxy groups of the cyclohexadienone component are disordered over two sets of sites with site occupancies of 0.6972 and 0.3028 . In the crystal, hydroxy $\mathrm{O}-\mathrm{H} \cdots \mathrm{O}$ hydrogen bonds to carbonyl O-atom acceptors form large centrosymmetric $R_{2}^{2}(36)$ cyclic dimers, which are further extended into supramolecular onedimensional ribbon structures along [1̄11].

\section{Chemical context}

The fruit rind of M. malabarica (family: Myristicaceae) is popularly known as Rampatri in Mumbai, India. It is used as an exotic spice in various Indian cuisines and also as a phytomedicine for the treatment of various kinds of ailments (Forrest \& Heacock, 1972, and references therein). Its major pharmacological activities are credited with hepatoprotective (Morita et al., 2003), anti-carcinogenic (Patro et al., 2010; Maity et al., 2012), anti-leishmanial (Sen et al., 2007), antiulceral (Banerjee et al., 2007; Banerjee et al., 2008), antiproliferative (Manna et al., 2012, 2015, 2016; Tyagi et al., 2014), anti-inflammatory (Maity et al., 2012), anti-quorum sensing (Chong et al., 2011) and anti-thrombotic (Olajide et al., 1999; Patro et al., 2005, 2010) properties and it is found as a constituent in many ayurvedic preparations such as Pasupasi. Previous phytochemical investigations of the fruit rind of $M$. malabarica revealed the presence of four novel diaryl nonanoids named as malabaricones A-D (Purushothaman et al., 1977) and aryl tetradecanoid (Bauri et al., 2016). In addition, a lignan malabaricanol $\mathrm{A}$ and an isoflavone have been isolated from the heart wood of this plant (Purushothaman et al., 1974; Talukdar et al., 2000). A detailed phytochemical investigation of a methanol extract of the fruit rind of M. malabarica has been carried out. We have isolated a new type of molecule named as an aryl cyclohexyl nonanoid, the title compound $\mathrm{C}_{21} \mathrm{H}_{26} \mathrm{O}_{5}$, as a very minor constituent in addition to the 


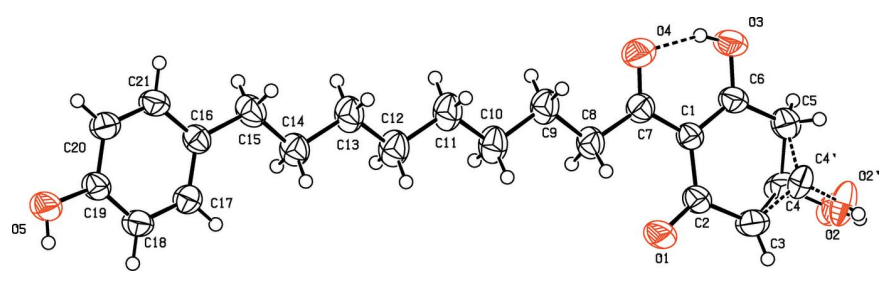

Figure 1

The molecular structure of the title compound, showing the atom labeling and displacement ellipsoids drawn at the $50 \%$ probability level. The disordered hydroxy group $\left(\mathrm{C} 4-\mathrm{O} 2-\mathrm{H} 2 A\right.$ and $\left.\mathrm{C} 2^{\prime}-\mathrm{O} 2^{\prime}-\mathrm{H} 2 B\right)$ is also shown, together with the intramolecular $\mathrm{O}-\mathrm{H} \cdots \mathrm{O}$ hydrogen bond.

reported compounds malabaricones A-D and aryl tetradecanoid. This molecule has exhibited anti-proliferative activity against various cancer cell lines such as A431, U937, MOLT-3, A549 and A2780 by using MTT and western blotting assay (unpublished result). Therefore, based on experimental results, it may be inferred that this fruit rind of $M$. malabarica may be used as a health promoter, a natural remedy which can be prescribed as a botanical dietary supplement to patients who are suffering from these kinds of health problems. The structure of the title compound is reported herein.<smiles>O=C(CCCCCCCCc1ccc(O)cc1)C1=C(O)C=C(O)CC1=O</smiles>

\section{Structural commentary}

The title compound comprises three molecular components, a 4-hydroxyphenyl ring, a 3,5-dihydroxcyclohexa-2,5-dienone ring and a bridging nonanoyl moiety (Fig. 1). The cyclohexa-
Table 1

Hydrogen-bond geometry $\left(\AA{ }^{\circ}\right)$.

\begin{tabular}{lllll}
\hline$D-\mathrm{H} \cdots A$ & $D-\mathrm{H}$ & $\mathrm{H} \cdots A$ & $D \cdots A$ & $D-\mathrm{H} \cdots A$ \\
\hline $\mathrm{O}^{2}-\mathrm{H} 2 A \cdots \mathrm{O} 5^{\mathrm{i}}$ & 0.83 & 2.18 & $3.004(7)$ & 174 \\
$\mathrm{O}^{\prime}-\mathrm{H} 2 B \cdots 5^{\mathrm{i}}$ & 0.82 & 1.86 & $2.565(16)$ & 143 \\
$\mathrm{O}^{2}-\mathrm{H} 3 O \cdots \mathrm{O} 4$ & 0.86 & 1.64 & $2.440(3)$ & 153 \\
$\mathrm{O}^{4}-\mathrm{H} 5 O \cdots{ }^{\mathrm{ii}}$ & 0.83 & 1.86 & $2.687(3)$ & 172 \\
\hline
\end{tabular}

Symmetry codes: (i) $x+1, y-1, z+1$; (ii) $-x-1,-y+1,-z+1$.

dienone ring has a puckered conformation. There is an intramolecular $\mathrm{O} 3-\mathrm{H} \cdots \mathrm{O} 4_{\text {carbonyl }}$ bond enclosing an $S(6)$ ring motif, which aids in stabilizing the essentially planar overall molecular conformation [inter-ring dihedral angle $=$ $6.37(15)^{\circ}$ and r.m.s. deviation of fitted atoms $\left.=0.2549 \AA\right]$. The $\mathrm{C}, \mathrm{O}$ and $\mathrm{H}$ atoms associated with the second hydroxy group of the cyclohexadienone component are disordered over two sets of sites $\left(\mathrm{C} 4, \mathrm{O} 2, \mathrm{H} 2 A\right.$ and $\left(\mathrm{C}^{\prime}, \mathrm{O} 2^{\prime}, \mathrm{H} 2 B\right)$ with a siteoccupancy factor of 0.6972:0.3028.

\section{Supramolecular features}

In the crystal, the molecules are linked by hydroxy O5$\mathrm{H} \cdot \mathrm{OO} 1^{\text {ii }}$ hydrogen bonds to carbonyl O-atom acceptors (Table 1), forming a primary large centrosymmetric $R_{2}^{2}(36)$ cyclic dimer (Fig. 2). These dimers are, in turn, linked through the disordered $\mathrm{C} 4$ hydroxy group $\left[\mathrm{O} 2-\mathrm{H} \cdots \mathrm{O}^{\mathrm{i}}\right.$ and $\mathrm{O}^{\prime}-$ $\left.\mathrm{H}^{\prime} \cdots \mathrm{O}^{\mathrm{i}}\right]$, extending the structure into a one-dimensional ribbon along [1]1] (Fig. 3). No inter-ring $\pi-\pi$ interactions are present in the structure (minimum ring-centroid separation $=$ $5.66 \AA$ A).

\section{Database survey}

A search of the Cambridge Structural Database (CSD, Version 5.37, updates November, 2015; Groom et al., 2016) has

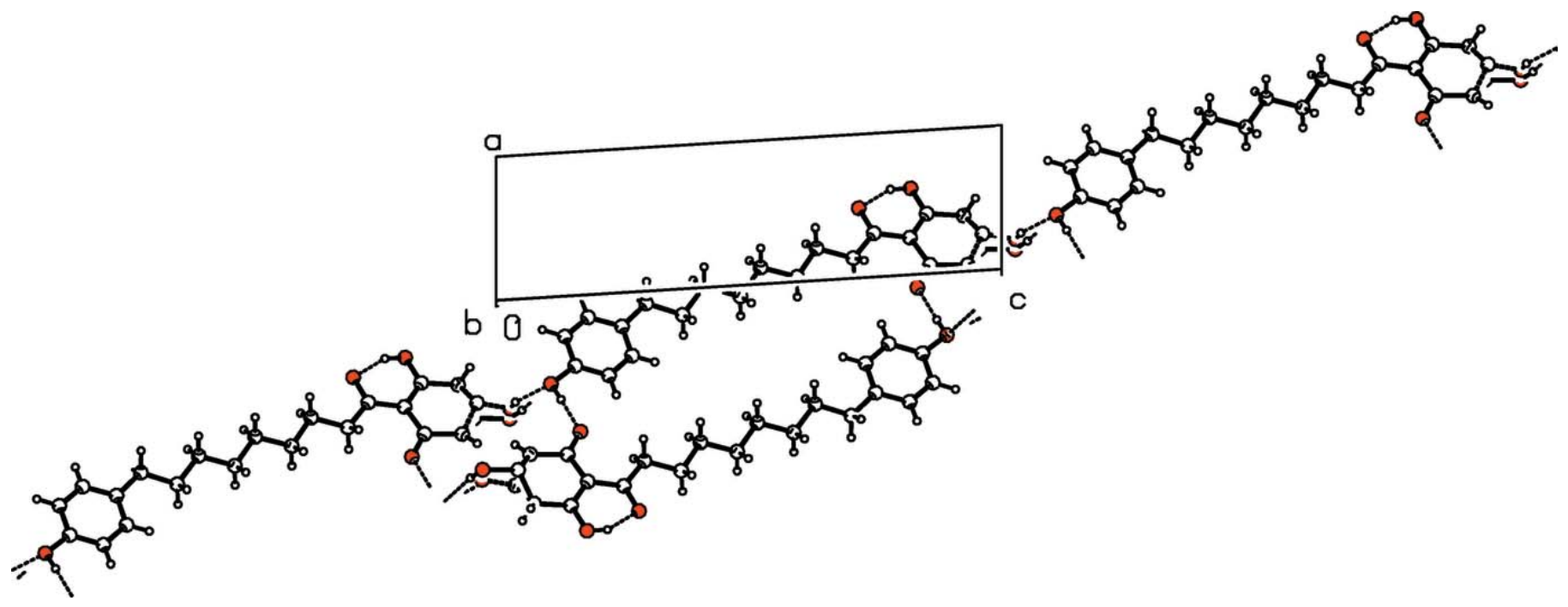

Figure 2

Centrosymmetric dimer formation in the crystal packing of the title compound, with intermolecular hydrogen bonds shown as dashed lines. 
registered two hits for the compounds found in $M$. malabarica: malabaricone-A (Bauri et al., 2006a) and malabaricone-C monohydrate (Bauri et al., 2006b), but no other examples were found resembling the title compound.

\section{Synthesis and crystallization}

The compound has been isolated as a very minor constituent from a methanol extraction of the fruit rind of $M$. malabarica by using $\mathrm{CC} / \mathrm{SiO}_{2}$ with gradient solvent elution with a binary mixture of solvent methanol and chloroform. Suitable crystals for X-ray data collection were obtained after recrystallization $(\times 3)$ from hexane:ethyl acetate $(4: 1)$, by slow evaporation at room temperature. The NMR spectroscopic analysis of the crystallized product has been interpreted as follows. ${ }^{1} \mathrm{H}$ NMR data (acetone- $\left.d_{6}, 200 \mathrm{MHz}\right): 8.80(s, b r s-\mathrm{OH}, 1 \mathrm{H}), 6.89(d d$, $\left.1 \mathrm{H}, J=8.2 \mathrm{~Hz}, \mathrm{H}-2^{\prime \prime} \& \mathrm{H}-6^{\prime \prime}, 2 \times \mathrm{Ar}-\mathrm{H}\right), 6.59(d d, 2 \mathrm{H}, J=$ $8.2 \mathrm{~Hz}, \mathrm{H}-3^{\prime \prime}$ \& H-5", $2 \times$ Ar-H), $4.20-4.15$ ( $\left.m, 1 \mathrm{H}, \mathrm{H}-6\right), 2.90$ $\left(d d, 2 \mathrm{H}, J=7.0 \mathrm{~Hz}, \mathrm{H}-2^{\prime}\right), 2.61-2.43(d d, 2 \mathrm{H}, J=2.20 \mathrm{~Hz}$ each, $\mathrm{H}-4), 2.39$ ( $d d, 2 \mathrm{H}, J=7.0 \mathrm{~Hz}, \mathrm{H}-9)$ 1.67-1.40 ( $m, 4 \mathrm{H}, \mathrm{H}-3^{\prime} \&$ $\left.\mathrm{H}-8^{\prime}\right), 1.19\left(s, 8 \mathrm{H}, 4 \times-\mathrm{CH}_{2} \mathrm{H}-4^{\prime} \mathrm{H}-5^{\prime}, \mathrm{H}-6^{\prime} \& \mathrm{H}-7^{\prime}\right) .{ }^{13} \mathrm{C} \mathrm{NMR}$ data $\left(50 \mathrm{MHz}\right.$,acetone- $\left.d_{6}\right): 205.69\left(\mathrm{C}-1^{\prime},>\mathrm{C}=\mathrm{O}\right), 198(\mathrm{C}-1$, $>\mathrm{C}=\mathrm{O}), 194$ (C-3 \& C-5, >C=C-OH), 156.20 (C-4", $\mathrm{Ar}-$ $\mathrm{C}-\mathrm{OH}), 129.94\left(\mathrm{C}-2^{\prime \prime}\right.$ \& C-6" $\left.{ }^{\prime \prime}, 2 \times \mathrm{Ar}-\mathrm{C}-\mathrm{H}\right), 116.6\left(\mathrm{C}-3^{\prime} \&\right.$ $\left.\mathrm{C}-5^{\prime}, \quad \mathrm{Ar}-\mathrm{C}-\mathrm{H}\right), 134.12 \quad(\mathrm{C}-6, \quad>\mathrm{C}=\mathrm{C}<), 113.60 \quad(\mathrm{C}-2$, $>\mathrm{C}=\mathrm{C}<), 47.58\left(\mathrm{C}-2^{\prime},-\mathrm{CH}_{2}-\mathrm{CO}-\right), 42.13\left(\mathrm{C}-9^{\prime}, \mathrm{Ar}-\mathrm{CH}_{2}\right)$, $40.57\left(\mathrm{C}-3^{\prime},-\mathrm{CH}_{2}-\mathrm{CH}_{2}\right), 35.56\left(\mathrm{C}-4^{\prime},-\mathrm{CH}_{2}-\mathrm{CH}_{2}-\right), 32.37$ $\left(\mathrm{C}-6^{\prime},-\mathrm{CH}_{2}-\mathrm{CH}_{2}\right), 30.19\left(\mathrm{C}-3^{\prime},-\mathrm{CH}_{2}-\mathrm{CH}_{2}-\right), 25.40$ (C-5', $-\mathrm{CH}_{2}$ ).

\section{Refinement}

Crystal data, data collection and structure refinement details are summarized in Table 2. The $\mathrm{H}$ atoms were positioned with idealized geometry using a riding model with aromatic $\mathrm{C}-\mathrm{H}=$ $0.93 \AA$ (aromatic) or $0.97 \AA$ (methylene). The $\mathrm{H}$ atoms of the $\mathrm{OH}$ groups were located in a difference map and were refined as riding on their parent $\mathrm{O}$ atoms. All $\mathrm{H}$ atoms were refined
Table 2

Experimental details.

\begin{tabular}{|c|c|}
\hline \multicolumn{2}{|l|}{ Crystal data } \\
\hline Chemical formula & $\mathrm{C}_{21} \mathrm{H}_{26} \mathrm{O}_{5}$ \\
\hline$M_{\mathrm{r}}$ & 358.42 \\
\hline Crystal system, space group & Triclinic, $P \overline{1}$ \\
\hline Temperature $(\mathrm{K})$ & 293 \\
\hline$a, b, c(\AA)$ & $5.6630(8), 8.707(1), 20.152(3)$ \\
\hline$\alpha, \beta, \gamma\left({ }^{\circ}\right)$ & $81.69(1), 86.48(1), 88.48(1)$ \\
\hline$V\left(\AA^{3}\right)$ & $981.2(2)$ \\
\hline$Z$ & 2 \\
\hline Radiation type & Мо $K \alpha$ \\
\hline$\mu\left(\mathrm{mm}^{-1}\right)$ & 0.09 \\
\hline Crystal size $(\mathrm{mm})$ & $0.48 \times 0.48 \times 0.20$ \\
\hline \multicolumn{2}{|l|}{ Data collection } \\
\hline Diffractometer & $\begin{array}{l}\text { Oxford Diffraction Xcalibur } \\
\text { diffractometer with Sapphire } \\
\text { CCD detector }\end{array}$ \\
\hline Absorption correction & $\begin{array}{l}\text { Multi-scan (CrysAlis RED; Oxford } \\
\quad \text { Diffraction, 2009) }\end{array}$ \\
\hline$T_{\min }, T_{\max }$ & $0.960,0.983$ \\
\hline $\begin{array}{l}\text { No. of measured, independent and } \\
\text { observed }[I>2 \sigma(I)] \text { reflections }\end{array}$ & $6013,3552,2638$ \\
\hline$R_{\text {int }}$ & 0.013 \\
\hline$(\sin \theta / \lambda)_{\max }\left(\AA^{-1}\right)$ & 0.602 \\
\hline \multicolumn{2}{|l|}{ Refinement } \\
\hline$R\left[F^{2}>2 \sigma\left(F^{2}\right)\right], w R\left(F^{2}\right), S$ & $0.071,0.178,1.10$ \\
\hline No. of reflections & 3552 \\
\hline No. of parameters & 254 \\
\hline No. of restraints & 3 \\
\hline $\mathrm{H}$-atom treatment & $\mathrm{H}$-atom parameters constrained \\
\hline$\Delta \rho_{\max }, \Delta \rho_{\min }\left(\mathrm{e} \AA^{-3}\right)$ & $0.36,-0.20$ \\
\hline
\end{tabular}

Computer programs: CrysAlis CCD and CrysAlis RED (Oxford Diffraction, 2009), SHELXS2014 (Sheldrick, 2008), SHELXL2014 (Sheldrick, 2015) and PLATON (Spek, 2009).

with isotropic displacement parameters set at $1.2 U_{\text {eq }}$ of the parent atom. The atoms $\mathrm{C} 4$ and $\mathrm{O} 2$ are disordered and were refined using a split model with site-occupancy factors 0.6972:0.3028. The corresponding bond distances in the disordered groups were restrained to be equal. The reflections $0 \overline{1} 14$ and 007 had poor disagreement with their calculated values and were omitted from the refinement.

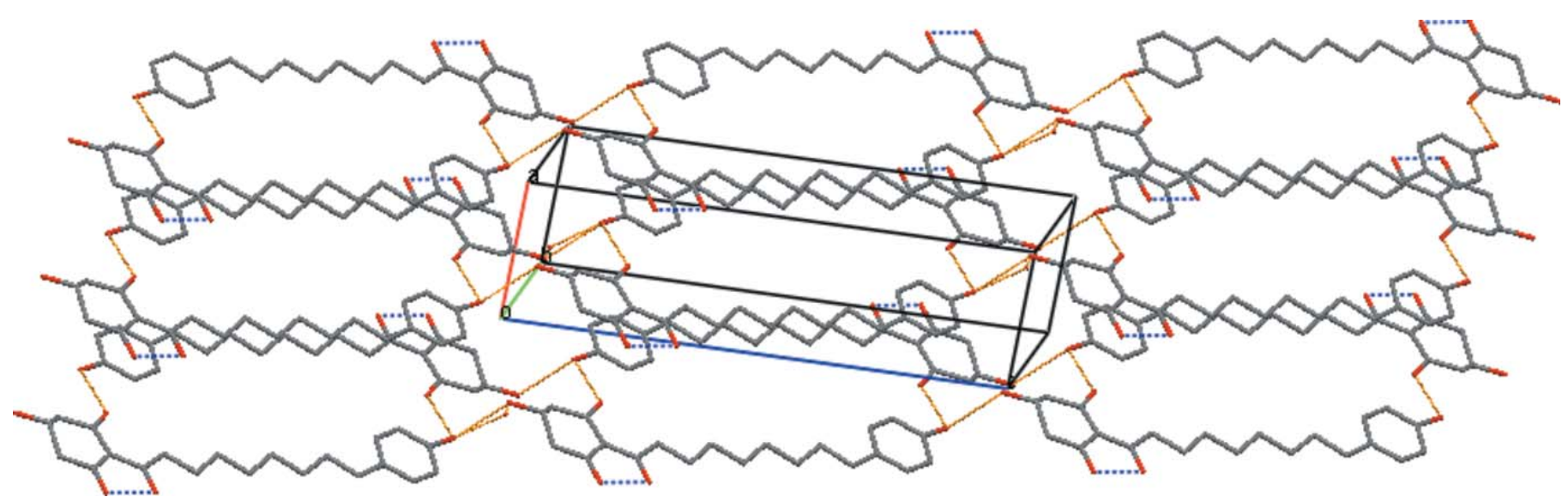

Figure 3

A view of the crystal packing in the unit cell, showing dimer extension into one-dimensional ribbons extending along [1 $\overline{1} 1]$. Blue- and orange-coloured dashed lines indicate the intra- and intermolecular $\mathrm{O}-\mathrm{H} \cdots \mathrm{O}$ hydrogen bonding. Only $\mathrm{H}$ atoms involved in hydrogen bonds are shown. 


\section{Acknowledgements}

The authors thank Professor Dr Hartmut Fuess, FG Strukturforschung, Material und Geowissenschaften, Technische Universität Darmstadt, Petersenstrasse 23, 64287 Darmstadt, and Professor Kingston, Department of Chemistry, M/C 0212, Virginia Polytechnic Institute and State University, Blacksburg, Virginia 24061, USA, for their kind co-operation to record X-ray diffraction data of the crystal, to provide diffractometer time and to carry out an antiproliferative bioassay against a cancer cell line.

\section{References}

Banerjee, D., Bauri, A. K., Guha, R. K., Bandyopadhyay, S. K. \& Chattopadhyay, S. (2008). Eur. J. Pharmacol. 578, 300-312.

Banerjee, D., Maity, B., Bauri, A. K., Bandyopadhyay, S. K. \& Chattopadhyay, S. (2007). J. Pharma. Pharmacol. 59, 15551565.

Bauri, A. K., Foro, S. \& Nhu Do, Q. N. (2016). IUCrData, 1, x160577.

Bauri, A. K., Foro, S., Lindner, H. J. \& Nayak, S. K. (2006a). Acta Cryst. E62, o1338-01339.

Bauri, A. K., Nayak, S. K., Foro, S. \& Lindner, H.-J. (2006b). Acta Cryst. E62, o2202-o2203.

Chong, Y. M., Yin, W. F., Ho, C. Y., Mustafa, M. S., Hadi, A. H. A., Awang, K., Narrima, P., Koh, C.-L., Appleton, D. R. \& Chan, K.-G. (2011). J. Nat. Prod. 74, 2261-2264.

Forrest, J. E. \& Heacock, R. A. (1972). Lloydia, 35, 440-449.

Groom, C. R., Bruno, I. J., Lightfoot, M. P. \& Ward, S. C. (2016). Acta Cryst. B72, 171-179.
Maity, B., Yadav, S. K., Patro, B. S., Tyagi, M., Bandyopadhyay, S. K. \& Chattopadhyay, S. (2012). Free Radic. Biol. Med. 52, 1680-1691.

Manna, A., De Sarkar, S., De, S., Bauri, A. K., Chattopadhyay, S. \& Chatterjee, M. (2015). Phytomedicine, 22, 713-723.

Manna, A., De Sarkar, S., De, S., Bauri, A. K., Chattopadhyay, S. \& Chatterjee, M. (2016). Int. Immunopharmacol. 39, 34-40.

Manna, A., Saha, P., Sarkar, A., Mukhopadhyay, D., Bauri, A. K., Kumar, D., Das, P., Chattopadhyay, S. \& Chatterjee, M. (2012). PLoS One, 45, 518-526.

Morita, T., Jinno, K., Kawagishi, H., Arimoto, Y., Suganuma, H., Inakuma, T. \& Sugiyama, K. (2003). J. Agric. Food Chem. 51, 15601565.

Olajide, O. A., Ajayi, F. F., Ekhelar, A. L., Awe, O. S., Makinde, J. M. \& Alada, A. R. (1999). Phytother. Res. 13, 344-345.

Oxford Diffraction (2009). CrysAlis CCD and CrysAlis RED. Oxford Diffraction Ltd, Yarnton, England.

Patro, B. S., Bauri, A. K., Mishra, S. \& Chattopadhyay, S. (2005). J. Agric. Food Chem. 53, 6912-6918.

Patro, B. S., Tyagi, M., Saha, J. \& Chattopadhyay, S. (2010). Bioorg. Med. Chem. 18, 7043-7051.

Purushothaman, K. K., Sarada, A. \& Connolly, J. D. (1974). Indian J. Chem. Sect. B, 23, 46-48.

Purushothaman, K. K., Sarada, A. \& Connolly, J. D. (1977). J. Chem. Soc. Perkin Trans. 1, pp. 587-588.

Sen, R., Bauri, A. K., Chattopadhyay, S. \& Chatterjee, M. (2007). Phytother. Res. 21, 592-595.

Sheldrick, G. M. (2008). Acta Cryst. A64, 112-122.

Sheldrick, G. M. (2015). Acta Cryst. C71, 3-8.

Spek, A. L. (2009). Acta Cryst. D65, 148-155.

Talukdar, A. C., Jain, N., De, S. \& Krishnamurty, H. G. (2000). Phytochemistry, 53, 155-157.

Tyagi, M., Bhattacharyya, R., Bauri, A. K., Patro, B. S. \& Chattopadhyay, S. (2014). Biochim. Biophys. Acta, 1840, 10141027. 


\section{supporting information}

Acta Cryst. (2016). E72, 1408-1411［https://doi.org/10.1107/S2056989016013797]

\section{Crystal structure of an aryl cyclohexyl nonanoid, an antiproliferative molecule isolated from the spice Myristica malabarica}

\section{Ajoy Kumar Bauri, Sabine Foro and Nhu Quynh Nguyen Do}

\section{Computing details}

Data collection: CrysAlis CCD (Oxford Diffraction, 2009); cell refinement: CrysAlis RED (Oxford Diffraction, 2009); data reduction: CrysAlis RED (Oxford Diffraction, 2009); program(s) used to solve structure: SHELXS2014 (Sheldrick, 2015); program(s) used to refine structure: SHELXL2014 (Sheldrick, 2015); molecular graphics: PLATON (Spek, 2009); software used to prepare material for publication: SHELXL2014 (Sheldrick, 2015).

3,5-Dihydroxy-2-[9-(4-hydroxyphenyl)nonanoyl]cyclohexa-2,4-dien-1-one

Crystal data

$\mathrm{C}_{21} \mathrm{H}_{26} \mathrm{O}_{5}$

$M_{r}=358.42$

Triclinic, $P \overline{1}$

$a=5.6630(8) \AA$

$b=8.707(1) \AA$

$c=20.152(3) \AA$

$\alpha=81.69(1)^{\circ}$

$\beta=86.48(1)^{\circ}$

$\gamma=88.48(1)^{\circ}$

$V=981.2(2) \AA^{3}$

\section{Data collection}

Oxford Diffraction Xcalibur diffractometer with Sapphire CCD detector

Radiation source: Enhance (Mo) X-ray source

Rotation method data acquisition using $\omega$ scans

Absorption correction: multi-scan

(CrysAlis RED; Oxford Diffraction, 2009)

$T_{\min }=0.960, T_{\max }=0.983$

6013 measured reflections

\section{Refinement}

Refinement on $F^{2}$

Least-squares matrix: full

$R\left[F^{2}>2 \sigma\left(F^{2}\right)\right]=0.071$

$w R\left(F^{2}\right)=0.178$

$S=1.10$

3552 reflections

254 parameters

3 restraints
$Z=2$

$F(000)=384$

$D_{\mathrm{x}}=1.213 \mathrm{Mg} \mathrm{m}^{-3}$

Mo $K \alpha$ radiation, $\lambda=0.71073 \AA$

Cell parameters from 2139 reflections

$\theta=2.9-27.7^{\circ}$

$\mu=0.09 \mathrm{~mm}^{-1}$

$T=293 \mathrm{~K}$

Prism, yellow

$0.48 \times 0.48 \times 0.20 \mathrm{~mm}$

3552 independent reflections

2638 reflections with $I>2 \sigma(I)$

$R_{\text {int }}=0.013$

$\theta_{\max }=25.4^{\circ}, \theta_{\min }=2.9^{\circ}$

$h=-6 \rightarrow 6$

$k=-10 \rightarrow 10$

$l=-21 \rightarrow 24$

Hydrogen site location: mixed

$\mathrm{H}$-atom parameters constrained

$w=1 /\left[\sigma^{2}\left(F_{\mathrm{o}}^{2}\right)+(0.0517 P)^{2}+0.9321 P\right]$

where $P=\left(F_{\mathrm{o}}{ }^{2}+2 F_{\mathrm{c}}{ }^{2}\right) / 3$

$(\Delta / \sigma)_{\max }<0.001$

$\Delta \rho_{\max }=0.36 \mathrm{e} \AA^{-3}$

$\Delta \rho_{\min }=-0.20 \mathrm{e}^{-3}$ 


\section{Special details}

Experimental. Absorption correction: CrysAlis RED (Oxford Diffraction, 2009) Empirical absorption correction using spherical harmonics, implemented in SCALE3 ABSPACK scaling algorithm.

Geometry. All esds (except the esd in the dihedral angle between two 1.s. planes) are estimated using the full covariance matrix. The cell esds are taken into account individually in the estimation of esds in distances, angles and torsion angles; correlations between esds in cell parameters are only used when they are defined by crystal symmetry. An approximate (isotropic) treatment of cell esds is used for estimating esds involving l.s. planes.

Fractional atomic coordinates and isotropic or equivalent isotropic displacement parameters $\left(\AA^{2}\right)$

\begin{tabular}{|c|c|c|c|c|c|}
\hline & $x$ & $y$ & $z$ & $U_{\text {iso }} * / U_{\text {eq }}$ & Occ. $(<1)$ \\
\hline $\mathrm{O} 1$ & $-0.0508(4)$ & $-0.0225(3)$ & $0.83338(11)$ & $0.0664(7)$ & \\
\hline $\mathrm{O} 2$ & $0.1863(14)$ & $-0.3525(8)$ & $1.0267(3)$ & $0.0655(19)$ & 0.6972 \\
\hline $\mathrm{H} 2 \mathrm{~A}$ & 0.2365 & -0.2936 & 1.0510 & $0.079 *$ & 0.6972 \\
\hline $\mathrm{O} 2^{\prime}$ & $0.254(4)$ & $-0.310(2)$ & $1.0277(7)$ & $0.069(5)$ & 0.3028 \\
\hline $\mathrm{H} 2 \mathrm{~B}$ & 0.2933 & -0.2259 & 1.0380 & $0.083^{*}$ & 0.3028 \\
\hline $\mathrm{O} 3$ & $0.6549(4)$ & $-0.3272(3)$ & $0.82020(11)$ & $0.0658(7)$ & \\
\hline $\mathrm{H} 3 \mathrm{O}$ & 0.6550 & -0.2796 & 0.7798 & $0.079^{*}$ & \\
\hline $\mathrm{O} 4$ & $0.5334(4)$ & $-0.1694(3)$ & $0.71742(10)$ & $0.0673(7)$ & \\
\hline O5 & $-0.5995(4)$ & $0.8647(2)$ & $0.10839(10)$ & $0.0617(6)$ & \\
\hline $\mathrm{H} 5 \mathrm{O}$ & -0.7128 & 0.9054 & 0.1283 & $0.074 *$ & \\
\hline $\mathrm{C} 1$ & $0.3066(5)$ & $-0.1679(3)$ & $0.81894(13)$ & $0.0427(6)$ & \\
\hline $\mathrm{C} 2$ & $0.1065(5)$ & $-0.1099(3)$ & $0.85825(14)$ & $0.0481(7)$ & \\
\hline $\mathrm{C} 3$ & $0.0917(6)$ & $-0.1598(4)$ & $0.93293(15)$ & $0.0742(11)$ & \\
\hline $\mathrm{H} 3$ & 0.0167 & -0.0988 & 0.9625 & $0.089 *$ & \\
\hline $\mathrm{C} 4$ & $0.2009(10)$ & $-0.3083(6)$ & $0.9562(2)$ & $0.0636(14)$ & 0.6972 \\
\hline $\mathrm{C} 4^{\prime}$ & $0.3050(18)$ & $-0.2280(11)$ & $0.9622(4)$ & $0.046(2)$ & 0.3028 \\
\hline $\mathrm{C} 5$ & $0.4429(6)$ & $-0.3327(4)$ & $0.92481(15)$ & $0.0605(9)$ & \\
\hline H5A & 0.5567 & -0.2819 & 0.9480 & $0.073^{*}$ & \\
\hline H5B & 0.4808 & -0.4430 & 0.9313 & $0.073^{*}$ & \\
\hline C6 & $0.4698(5)$ & $-0.2731(3)$ & $0.85166(14)$ & $0.0470(7)$ & \\
\hline $\mathrm{C} 7$ & $0.3503(5)$ & $-0.1185(3)$ & $0.74718(14)$ & $0.0467(7)$ & \\
\hline $\mathrm{C} 8$ & $0.1815(5)$ & $-0.0110(3)$ & $0.70682(14)$ & $0.0501(7)$ & \\
\hline H8A & 0.1566 & 0.0819 & 0.7279 & $0.060 *$ & \\
\hline H8B & 0.0303 & -0.0616 & 0.7084 & $0.060^{*}$ & \\
\hline $\mathrm{C} 9$ & $0.2634(6)$ & $0.0367(4)$ & $0.63382(14)$ & $0.0566(8)$ & \\
\hline H9A & 0.3025 & -0.0553 & 0.6131 & $0.068^{*}$ & \\
\hline H9B & 0.4049 & 0.0981 & 0.6314 & $0.068^{*}$ & \\
\hline $\mathrm{C} 10$ & $0.0701(6)$ & $0.1314(4)$ & $0.59556(15)$ & $0.0598(8)$ & \\
\hline H10A & -0.0672 & 0.0670 & 0.5959 & $0.072 *$ & \\
\hline H10B & 0.0231 & 0.2186 & 0.6187 & $0.072 *$ & \\
\hline C11 & $0.1480(6)$ & $0.1921(4)$ & $0.52332(15)$ & $0.0627(9)$ & \\
\hline H11A & 0.2042 & 0.1053 & 0.5010 & $0.075^{*}$ & \\
\hline H11B & 0.2796 & 0.2612 & 0.5232 & $0.075^{*}$ & \\
\hline $\mathrm{C} 12$ & $-0.0481(6)$ & $0.2792(4)$ & $0.48314(15)$ & $0.0614(9)$ & \\
\hline $\mathrm{H} 12 \mathrm{~A}$ & -0.1808 & 0.2108 & 0.4836 & $0.074 *$ & \\
\hline H12B & -0.1027 & 0.3672 & 0.5048 & $0.074 *$ & \\
\hline $\mathrm{C} 13$ & $0.0332(6)$ & $0.3360(4)$ & $0.41130(15)$ & $0.0599(8)$ & \\
\hline
\end{tabular}




$\begin{array}{lllll}\text { H13A } & 0.0865 & 0.2474 & 0.3899 & 0.072^{*} \\ \text { H13B } & 0.1679 & 0.4026 & 0.4113 & 0.072^{*} \\ \text { C14 } & -0.1574(6) & 0.4262(3) & 0.36914(14) & 0.0528(7) \\ \text { H14A } & -0.2963 & 0.3627 & 0.3705 & 0.063^{*} \\ \text { H14B } & -0.2036 & 0.5197 & 0.3880 & 0.063^{*} \\ \text { C15 } & -0.0638(5) & 0.4693(4) & 0.29700(15) & 0.0553(8) \\ \text { H15A } & -0.0314 & 0.3738 & 0.2783 & 0.066^{*} \\ \text { H15B } & 0.0863 & 0.5201 & 0.2975 & 0.066^{*} \\ \text { C16 } & -0.2175(5) & 0.5731(3) & 0.24928(14) & 0.0433(6) \\ \text { C17 } & -0.4281(5) & 0.6465(3) & 0.26883(14) & 0.0486(7) \\ \text { H17 } & -0.4819 & 0.6297 & 0.3138 & 0.058^{*} \\ \text { C18 } & -0.5602(5) & 0.7439(3) & 0.22330(14) & 0.0492(7) \\ \text { H18 } & -0.7001 & 0.7906 & 0.2376 & 0.059^{*} \\ \text { C19 } & -0.4795(5) & 0.7697(3) & 0.15660(14) & 0.0458(7) \\ \text { C20 } & -0.2681(5) & 0.6987(3) & 0.13615(14) & 0.0528(8) \\ \text { H20 } & -0.2128 & 0.7169 & 0.0913 & 0.063^{*} \\ \text { C21 } & -0.1409(5) & 0.6019(3) & 0.18188(14) & 0.0502(7) \\ \text { H21 } & -0.0015 & 0.5550 & 0.1673 & 0.060^{*}\end{array}$

Atomic displacement parameters $\left(\AA^{2}\right)$

\begin{tabular}{|c|c|c|c|c|c|c|}
\hline & $U^{11}$ & $U^{22}$ & $U^{33}$ & $U^{12}$ & $U^{13}$ & $U^{23}$ \\
\hline $\mathrm{O} 1$ & $0.0639(14)$ & $0.0742(15)$ & $0.0563(13)$ & $0.0366(12)$ & $-0.0044(10)$ & $0.0007(11)$ \\
\hline $\mathrm{O} 2$ & $0.082(5)$ & $0.068(4)$ & $0.043(2)$ & $0.013(3)$ & $0.005(2)$ & $-0.001(2)$ \\
\hline $\mathrm{O} 2^{\prime}$ & 0.107 (13) & $0.067(9)$ & $0.032(5)$ & $-0.021(6)$ & $-0.013(5)$ & $0.004(5)$ \\
\hline $\mathrm{O} 3$ & $0.0579(13)$ & $0.0792(15)$ & $0.0541(12)$ & $0.0328(12)$ & $0.0045(10)$ & 0.0024 \\
\hline $\mathrm{O} 4$ & $0.0666(15)$ & $0.0829(16)$ & $0.0474(12)$ & $0.0210(12)$ & $0.0073(10)$ & $-0.0015(11)$ \\
\hline $\mathrm{O} 5$ & $0.0625(14)$ & $0.0668(14)$ & $0.0496(12)$ & $0.0272(11)$ & $-0.0018(10)$ & $0.0066(10)$ \\
\hline $\mathrm{C} 1$ & $0.0426(15)$ & $0.0434(15)$ & $0.0404(14)$ & $0.0073(12)$ & $-0.0031(11)$ & $-0.0018(11)$ \\
\hline $\mathrm{C} 2$ & $0.0477(16)$ & $0.0482(16)$ & $0.0463(16)$ & $0.0125(13)$ & $-0.0043(13)$ & $-0.0017(13)$ \\
\hline $\mathrm{C} 3$ & $0.073(2)$ & $0.099(3)$ & $0.0426(17)$ & $0.044(2)$ & $0.0076(16)$ & $0.0028(17)$ \\
\hline $\mathrm{C} 4$ & $0.078(4)$ & $0.070(3)$ & $0.036(2)$ & $0.032(3)$ & $0.010(2)$ & $0.006(2)$ \\
\hline $\mathrm{C} 4^{\prime}$ & $0.068(7)$ & $0.040(5)$ & $0.030(5)$ & $-0.002(5)$ & $-0.007(4)$ & -0.005 \\
\hline $\mathrm{C} 5$ & $0.062(2)$ & $0.068(2)$ & $0.0464(17)$ & $0.0210(16)$ & $-0.0074(14)$ & $0.0038(15)$ \\
\hline C6 & $0.0430(16)$ & $0.0492(16)$ & $0.0470(16)$ & $0.0106(13)$ & $-0.0005(12)$ & $-0.0041(13)$ \\
\hline $\mathrm{C} 7$ & $0.0523(17)$ & $0.0438(15)$ & $0.0437(15)$ & $0.0036(13)$ & $-0.0044(13)$ & $-0.0053(12)$ \\
\hline $\mathrm{C} 8$ & $0.0565(18)$ & $0.0486(16)$ & $0.0429(15)$ & $0.0030(14)$ & $-0.0080(13)$ & $0.0019(12)$ \\
\hline C9 & $0.071(2)$ & $0.0545(18)$ & $0.0433(16)$ & $0.0027(15)$ & $-0.0105(14)$ & $0.0005(13)$ \\
\hline $\mathrm{C} 10$ & $0.078(2)$ & $0.0532(18)$ & 0.0467 (17) & $0.0009(16)$ & $-0.0139(15)$ & $0.0031(14)$ \\
\hline C11 & $0.081(2)$ & 0.0580 (19) & $0.0471(17)$ & $0.0033(17)$ & $-0.0151(16)$ & $0.0034(14)$ \\
\hline $\mathrm{C} 12$ & $0.081(2)$ & $0.0522(18)$ & $0.0492(17)$ & $0.0053(16)$ & $-0.0117(16)$ & $0.0014(14)$ \\
\hline $\mathrm{C} 13$ & $0.074(2)$ & $0.0544(18)$ & 0.0488 (17) & $0.0063(16)$ & $-0.0148(15)$ & $0.0037(14)$ \\
\hline $\mathrm{C} 14$ & 0.0605 (19) & $0.0465(16)$ & $0.0492(17)$ & $0.0035(14)$ & $-0.0075(14)$ & $0.0018(13)$ \\
\hline $\mathrm{C} 15$ & $0.0510(18)$ & $0.0560(18)$ & $0.0542(18)$ & $0.0050(14)$ & $-0.0073(14)$ & $0.0082(14)$ \\
\hline $\mathrm{C} 16$ & $0.0428(15)$ & $0.0400(14)$ & $0.0455(15)$ & $0.0013(12)$ & $-0.0052(12)$ & $0.0001(12)$ \\
\hline $\mathrm{C} 17$ & $0.0486(17)$ & $0.0539(17)$ & $0.0400(15)$ & $0.0034(13)$ & $0.0017(12)$ & $0.0012(13)$ \\
\hline C18 & $0.0437(16)$ & $0.0503(17)$ & $0.0518(17)$ & $0.0084(13)$ & $0.0025(13)$ & $-0.0048(13)$ \\
\hline C19 & $0.0462(16)$ & $0.0431(15)$ & $0.0461(15)$ & $0.0081(13)$ & $-0.0040(12)$ & $-0.0003(12)$ \\
\hline
\end{tabular}




\begin{tabular}{lllllll}
$\mathrm{C} 20$ & $0.0544(18)$ & $0.0581(18)$ & $0.0424(15)$ & $0.0147(15)$ & $0.0031(13)$ & $-0.0009(13)$ \\
$\mathrm{C} 21$ & $0.0461(17)$ & $0.0520(17)$ & $0.0504(16)$ & $0.0140(13)$ & $0.0004(13)$ & $-0.0039(13)$ \\
\hline
\end{tabular}

Geometric parameters $\left(\AA,{ }^{\circ}\right)$

\begin{tabular}{|c|c|c|c|}
\hline $\mathrm{O} 1-\mathrm{C} 2$ & $1.238(3)$ & $\mathrm{C} 10-\mathrm{C} 11$ & $1.518(4)$ \\
\hline $\mathrm{O} 2-\mathrm{C} 4$ & $1.415(7)$ & $\mathrm{C} 10-\mathrm{H} 10 \mathrm{~A}$ & 0.9700 \\
\hline $\mathrm{O} 2-\mathrm{H} 2 \mathrm{~A}$ & 0.8256 & $\mathrm{C} 10-\mathrm{H} 10 \mathrm{~B}$ & 0.9700 \\
\hline $\mathrm{O} 2^{\prime}-\mathrm{H} 2 \mathrm{~B}$ & 0.8247 & $\mathrm{C} 11-\mathrm{C} 12$ & $1.534(4)$ \\
\hline $\mathrm{O} 3-\mathrm{C} 6$ & $1.305(3)$ & $\mathrm{C} 11-\mathrm{H} 11 \mathrm{~A}$ & 0.9700 \\
\hline $\mathrm{O} 3-\mathrm{H} 3 \mathrm{O}$ & 0.8588 & C11-H11B & 0.9700 \\
\hline $\mathrm{O} 4-\mathrm{C} 7$ & $1.268(3)$ & $\mathrm{C} 12-\mathrm{C} 13$ & 1.509 (4) \\
\hline $\mathrm{O} 5-\mathrm{C} 19$ & $1.382(3)$ & $\mathrm{C} 12-\mathrm{H} 12 \mathrm{~A}$ & 0.9700 \\
\hline $\mathrm{O} 5-\mathrm{H} 5 \mathrm{O}$ & 0.8342 & $\mathrm{C} 12-\mathrm{H} 12 \mathrm{~B}$ & 0.9700 \\
\hline $\mathrm{C} 1-\mathrm{C} 6$ & $1.408(4)$ & $\mathrm{C} 13-\mathrm{C} 14$ & $1.543(4)$ \\
\hline $\mathrm{C} 1-\mathrm{C} 7$ & $1.456(4)$ & $\mathrm{C} 13-\mathrm{H} 13 \mathrm{~A}$ & 0.9700 \\
\hline $\mathrm{C} 1-\mathrm{C} 2$ & $1.463(4)$ & C13-H13B & 0.9700 \\
\hline $\mathrm{C} 2-\mathrm{C} 3$ & $1.503(4)$ & $\mathrm{C} 14-\mathrm{C} 15$ & $1.514(4)$ \\
\hline $\mathrm{C} 3-\mathrm{C} 4$ & $1.446(5)$ & $\mathrm{C} 14-\mathrm{H} 14 \mathrm{~A}$ & 0.9700 \\
\hline $\mathrm{C} 3-\mathrm{C}^{\prime}$ & $1.452(10)$ & C14-H14B & 0.9700 \\
\hline $\mathrm{C} 3-\mathrm{H} 3$ & 0.9300 & $\mathrm{C} 15-\mathrm{C} 16$ & $1.519(4)$ \\
\hline $\mathrm{C} 4-\mathrm{C} 5$ & $1.497(5)$ & $\mathrm{C} 15-\mathrm{H} 15 \mathrm{~A}$ & 0.9700 \\
\hline $\mathrm{C} 4^{\prime}-\mathrm{C} 5$ & $1.449(9)$ & C15-H15B & 0.9700 \\
\hline $\mathrm{C} 5-\mathrm{C} 6$ & $1.490(4)$ & $\mathrm{C} 16-\mathrm{C} 21$ & $1.390(4)$ \\
\hline $\mathrm{C} 5-\mathrm{H} 5 \mathrm{~A}$ & 0.9700 & $\mathrm{C} 16-\mathrm{C} 17$ & $1.400(4)$ \\
\hline C5-H5B & 0.9700 & $\mathrm{C} 17-\mathrm{C} 18$ & $1.395(4)$ \\
\hline $\mathrm{C} 7-\mathrm{C} 8$ & $1.510(4)$ & C17-H17 & 0.9300 \\
\hline $\mathrm{C} 8-\mathrm{C} 9$ & $1.517(4)$ & $\mathrm{C} 18-\mathrm{C} 19$ & $1.382(4)$ \\
\hline $\mathrm{C} 8-\mathrm{H} 8 \mathrm{~A}$ & 0.9700 & C18-H18 & 0.9300 \\
\hline $\mathrm{C} 8-\mathrm{H} 8 \mathrm{~B}$ & 0.9700 & $\mathrm{C} 19-\mathrm{C} 20$ & $1.400(4)$ \\
\hline $\mathrm{C} 9-\mathrm{C} 10$ & $1.531(4)$ & $\mathrm{C} 20-\mathrm{C} 21$ & $1.379(4)$ \\
\hline C9-H9A & 0.9700 & $\mathrm{C} 20-\mathrm{H} 20$ & 0.9300 \\
\hline C9-H9B & 0.9700 & $\mathrm{C} 21-\mathrm{H} 21$ & 0.9300 \\
\hline $\mathrm{C} 4-\mathrm{O} 2-\mathrm{H} 2 \mathrm{~A}$ & 119.3 & $\mathrm{C} 10-\mathrm{C} 11-\mathrm{C} 12$ & $113.8(3)$ \\
\hline $\mathrm{C} 6-\mathrm{O} 3-\mathrm{H} 3 \mathrm{O}$ & 105.4 & $\mathrm{C} 10-\mathrm{C} 11-\mathrm{H} 11 \mathrm{~A}$ & 108.8 \\
\hline $\mathrm{C} 19-\mathrm{O} 5-\mathrm{H} 5 \mathrm{O}$ & 107.1 & $\mathrm{C} 12-\mathrm{C} 11-\mathrm{H} 11 \mathrm{~A}$ & 108.8 \\
\hline $\mathrm{C} 6-\mathrm{C} 1-\mathrm{C} 7$ & $117.7(2)$ & $\mathrm{C} 10-\mathrm{C} 11-\mathrm{H} 11 \mathrm{~B}$ & 108.8 \\
\hline $\mathrm{C} 6-\mathrm{C} 1-\mathrm{C} 2$ & $119.2(2)$ & $\mathrm{C} 12-\mathrm{C} 11-\mathrm{H} 11 \mathrm{~B}$ & 108.8 \\
\hline $\mathrm{C} 7-\mathrm{C} 1-\mathrm{C} 2$ & $123.1(2)$ & $\mathrm{H} 11 \mathrm{~A}-\mathrm{C} 11-\mathrm{H} 11 \mathrm{~B}$ & 107.7 \\
\hline $\mathrm{O} 1-\mathrm{C} 2-\mathrm{C} 1$ & $123.6(3)$ & $\mathrm{C} 13-\mathrm{C} 12-\mathrm{C} 11$ & $112.6(3)$ \\
\hline $\mathrm{O} 1-\mathrm{C} 2-\mathrm{C} 3$ & $118.4(3)$ & $\mathrm{C} 13-\mathrm{C} 12-\mathrm{H} 12 \mathrm{~A}$ & 109.1 \\
\hline $\mathrm{C} 1-\mathrm{C} 2-\mathrm{C} 3$ & $118.0(2)$ & $\mathrm{C} 11-\mathrm{C} 12-\mathrm{H} 12 \mathrm{~A}$ & 109.1 \\
\hline $\mathrm{C} 4-\mathrm{C} 3-\mathrm{C} 2$ & $116.1(3)$ & $\mathrm{C} 13-\mathrm{C} 12-\mathrm{H} 12 \mathrm{~B}$ & 109.1 \\
\hline $\mathrm{C} 4^{\prime}-\mathrm{C} 3-\mathrm{C} 2$ & $116.5(4)$ & $\mathrm{C} 11-\mathrm{C} 12-\mathrm{H} 12 \mathrm{~B}$ & 109.1 \\
\hline $\mathrm{C} 4-\mathrm{C} 3-\mathrm{H} 3$ & 122.0 & $\mathrm{H} 12 \mathrm{~A}-\mathrm{C} 12-\mathrm{H} 12 \mathrm{~B}$ & 107.8 \\
\hline $\mathrm{C} 2-\mathrm{C} 3-\mathrm{H} 3$ & 122.0 & $\mathrm{C} 12-\mathrm{C} 13-\mathrm{C} 14$ & $114.5(3)$ \\
\hline $\mathrm{O} 2-\mathrm{C} 4-\mathrm{C} 3$ & $115.1(5)$ & $\mathrm{C} 12-\mathrm{C} 13-\mathrm{H} 13 \mathrm{~A}$ & 108.6 \\
\hline
\end{tabular}




\begin{tabular}{|c|c|c|c|}
\hline $\mathrm{O} 2-\mathrm{C} 4-\mathrm{C} 5$ & $113.0(5)$ & $\mathrm{C} 14-\mathrm{C} 13-\mathrm{H} 13 \mathrm{~A}$ & 108.6 \\
\hline $\mathrm{C} 3-\mathrm{C} 4-\mathrm{C} 5$ & $114.4(4)$ & $\mathrm{C} 12-\mathrm{C} 13-\mathrm{H} 13 \mathrm{~B}$ & 108.6 \\
\hline $\mathrm{C} 5-\mathrm{C} 4^{\prime}-\mathrm{C} 3$ & $117.1(6)$ & $\mathrm{C} 14-\mathrm{C} 13-\mathrm{H} 13 \mathrm{~B}$ & 108.6 \\
\hline $\mathrm{C} 4{ }^{\prime}-\mathrm{C} 5-\mathrm{C} 6$ & $112.4(4)$ & $\mathrm{H} 13 \mathrm{~A}-\mathrm{C} 13-\mathrm{H} 13 \mathrm{~B}$ & 107.6 \\
\hline $\mathrm{C} 6-\mathrm{C} 5-\mathrm{C} 4$ & $114.3(3)$ & $\mathrm{C} 15-\mathrm{C} 14-\mathrm{C} 13$ & $110.4(3)$ \\
\hline $\mathrm{C} 6-\mathrm{C} 5-\mathrm{H} 5 \mathrm{~A}$ & 108.7 & $\mathrm{C} 15-\mathrm{C} 14-\mathrm{H} 14 \mathrm{~A}$ & 109.6 \\
\hline $\mathrm{C} 4-\mathrm{C} 5-\mathrm{H} 5 \mathrm{~A}$ & 108.7 & $\mathrm{C} 13-\mathrm{C} 14-\mathrm{H} 14 \mathrm{~A}$ & 109.6 \\
\hline $\mathrm{C} 6-\mathrm{C} 5-\mathrm{H} 5 \mathrm{~B}$ & 108.7 & $\mathrm{C} 15-\mathrm{C} 14-\mathrm{H} 14 \mathrm{~B}$ & 109.6 \\
\hline $\mathrm{C} 4-\mathrm{C} 5-\mathrm{H} 5 \mathrm{~B}$ & 108.7 & $\mathrm{C} 13-\mathrm{C} 14-\mathrm{H} 14 \mathrm{~B}$ & 109.6 \\
\hline $\mathrm{H} 5 \mathrm{~A}-\mathrm{C} 5-\mathrm{H} 5 \mathrm{~B}$ & 107.6 & $\mathrm{H} 14 \mathrm{~A}-\mathrm{C} 14-\mathrm{H} 14 \mathrm{~B}$ & 108.1 \\
\hline $\mathrm{O} 3-\mathrm{C} 6-\mathrm{C} 1$ & $122.7(3)$ & $\mathrm{C} 14-\mathrm{C} 15-\mathrm{C} 16$ & $118.1(3)$ \\
\hline $\mathrm{O} 3-\mathrm{C} 6-\mathrm{C} 5$ & $114.7(2)$ & $\mathrm{C} 14-\mathrm{C} 15-\mathrm{H} 15 \mathrm{~A}$ & 107.8 \\
\hline $\mathrm{C} 1-\mathrm{C} 6-\mathrm{C} 5$ & $122.6(2)$ & $\mathrm{C} 16-\mathrm{C} 15-\mathrm{H} 15 \mathrm{~A}$ & 107.8 \\
\hline $\mathrm{O} 4-\mathrm{C} 7-\mathrm{C} 1$ & $119.1(2)$ & $\mathrm{C} 14-\mathrm{C} 15-\mathrm{H} 15 \mathrm{~B}$ & 107.8 \\
\hline $\mathrm{O} 4-\mathrm{C} 7-\mathrm{C} 8$ & $119.0(2)$ & $\mathrm{C} 16-\mathrm{C} 15-\mathrm{H} 15 \mathrm{~B}$ & 107.8 \\
\hline $\mathrm{C} 1-\mathrm{C} 7-\mathrm{C} 8$ & $122.0(2)$ & $\mathrm{H} 15 \mathrm{~A}-\mathrm{C} 15-\mathrm{H} 15 \mathrm{~B}$ & 107.1 \\
\hline $\mathrm{C} 7-\mathrm{C} 8-\mathrm{C} 9$ & $114.7(3)$ & $\mathrm{C} 21-\mathrm{C} 16-\mathrm{C} 17$ & $117.4(2)$ \\
\hline $\mathrm{C} 7-\mathrm{C} 8-\mathrm{H} 8 \mathrm{~A}$ & 108.6 & $\mathrm{C} 21-\mathrm{C} 16-\mathrm{C} 15$ & $118.1(3)$ \\
\hline $\mathrm{C} 9-\mathrm{C} 8-\mathrm{H} 8 \mathrm{~A}$ & 108.6 & $\mathrm{C} 17-\mathrm{C} 16-\mathrm{C} 15$ & $124.5(3)$ \\
\hline $\mathrm{C} 7-\mathrm{C} 8-\mathrm{H} 8 \mathrm{~B}$ & 108.6 & $\mathrm{C} 18-\mathrm{C} 17-\mathrm{C} 16$ & $122.4(3)$ \\
\hline $\mathrm{C} 9-\mathrm{C} 8-\mathrm{H} 8 \mathrm{~B}$ & 108.6 & $\mathrm{C} 18-\mathrm{C} 17-\mathrm{H} 17$ & 118.8 \\
\hline $\mathrm{H} 8 \mathrm{~A}-\mathrm{C} 8-\mathrm{H} 8 \mathrm{~B}$ & 107.6 & $\mathrm{C} 16-\mathrm{C} 17-\mathrm{H} 17$ & 118.8 \\
\hline $\mathrm{C} 8-\mathrm{C} 9-\mathrm{C} 10$ & $110.7(3)$ & $\mathrm{C} 19-\mathrm{C} 18-\mathrm{C} 17$ & $118.8(3)$ \\
\hline $\mathrm{C} 8-\mathrm{C} 9-\mathrm{H} 9 \mathrm{~A}$ & 109.5 & $\mathrm{C} 19-\mathrm{C} 18-\mathrm{H} 18$ & 120.6 \\
\hline $\mathrm{C} 10-\mathrm{C} 9-\mathrm{H} 9 \mathrm{~A}$ & 109.5 & $\mathrm{C} 17-\mathrm{C} 18-\mathrm{H} 18$ & 120.6 \\
\hline $\mathrm{C} 8-\mathrm{C} 9-\mathrm{H} 9 \mathrm{~B}$ & 109.5 & $\mathrm{O} 5-\mathrm{C} 19-\mathrm{C} 18$ & $122.4(2)$ \\
\hline $\mathrm{C} 10-\mathrm{C} 9-\mathrm{H} 9 \mathrm{~B}$ & 109.5 & $\mathrm{O} 5-\mathrm{C} 19-\mathrm{C} 20$ & $117.9(2)$ \\
\hline $\mathrm{H} 9 \mathrm{~A}-\mathrm{C} 9-\mathrm{H} 9 \mathrm{~B}$ & 108.1 & $\mathrm{C} 18-\mathrm{C} 19-\mathrm{C} 20$ & $119.7(2)$ \\
\hline $\mathrm{C} 11-\mathrm{C} 10-\mathrm{C} 9$ & $113.2(3)$ & $\mathrm{C} 21-\mathrm{C} 20-\mathrm{C} 19$ & $120.6(3)$ \\
\hline $\mathrm{C} 11-\mathrm{C} 10-\mathrm{H} 10 \mathrm{~A}$ & 108.9 & $\mathrm{C} 21-\mathrm{C} 20-\mathrm{H} 20$ & 119.7 \\
\hline $\mathrm{C} 9-\mathrm{C} 10-\mathrm{H} 10 \mathrm{~A}$ & 108.9 & $\mathrm{C} 19-\mathrm{C} 20-\mathrm{H} 20$ & 119.7 \\
\hline $\mathrm{C} 11-\mathrm{C} 10-\mathrm{H} 10 \mathrm{~B}$ & 108.9 & $\mathrm{C} 20-\mathrm{C} 21-\mathrm{C} 16$ & $121.2(3)$ \\
\hline $\mathrm{C} 9-\mathrm{C} 10-\mathrm{H} 10 \mathrm{~B}$ & 108.9 & $\mathrm{C} 20-\mathrm{C} 21-\mathrm{H} 21$ & 119.4 \\
\hline $\mathrm{H} 10 \mathrm{~A}-\mathrm{C} 10-\mathrm{H} 10 \mathrm{~B}$ & 107.8 & $\mathrm{C} 16-\mathrm{C} 21-\mathrm{H} 21$ & 119.4 \\
\hline $\mathrm{C} 6-\mathrm{C} 1-\mathrm{C} 2-\mathrm{O} 1$ & $-176.9(3)$ & $\mathrm{C} 6-\mathrm{C} 1-\mathrm{C} 7-\mathrm{C} 8$ & $177.6(3)$ \\
\hline $\mathrm{C} 7-\mathrm{C} 1-\mathrm{C} 2-\mathrm{O} 1$ & $5.0(5)$ & $\mathrm{C} 2-\mathrm{C} 1-\mathrm{C} 7-\mathrm{C} 8$ & $-4.3(4)$ \\
\hline $\mathrm{C} 6-\mathrm{C} 1-\mathrm{C} 2-\mathrm{C} 3$ & $3.0(4)$ & $\mathrm{O} 4-\mathrm{C} 7-\mathrm{C} 8-\mathrm{C} 9$ & $-5.2(4)$ \\
\hline $\mathrm{C} 7-\mathrm{C} 1-\mathrm{C} 2-\mathrm{C} 3$ & $-175.1(3)$ & $\mathrm{C} 1-\mathrm{C} 7-\mathrm{C} 8-\mathrm{C} 9$ & $176.1(3)$ \\
\hline $\mathrm{O} 1-\mathrm{C} 2-\mathrm{C} 3-\mathrm{C} 4$ & $152.2(4)$ & $\mathrm{C} 7-\mathrm{C} 8-\mathrm{C} 9-\mathrm{C} 10$ & $174.2(3)$ \\
\hline $\mathrm{C} 1-\mathrm{C} 2-\mathrm{C} 3-\mathrm{C} 4$ & $-27.6(5)$ & $\mathrm{C} 8-\mathrm{C} 9-\mathrm{C} 10-\mathrm{C} 11$ & $176.0(3)$ \\
\hline $\mathrm{O} 1-\mathrm{C} 2-\mathrm{C} 3-\mathrm{C} 4^{\prime}$ & $-164.8(5)$ & $\mathrm{C} 9-\mathrm{C} 10-\mathrm{C} 11-\mathrm{C} 12$ & $176.6(3)$ \\
\hline $\mathrm{C} 1-\mathrm{C} 2-\mathrm{C} 3-\mathrm{C} 4{ }^{\prime}$ & $15.4(7)$ & $\mathrm{C} 10-\mathrm{C} 11-\mathrm{C} 12-\mathrm{C} 13$ & $-179.1(3)$ \\
\hline $\mathrm{C} 2-\mathrm{C} 3-\mathrm{C} 4-\mathrm{O} 2$ & $179.6(5)$ & $\mathrm{C} 11-\mathrm{C} 12-\mathrm{C} 13-\mathrm{C} 14$ & $-179.2(3)$ \\
\hline $\mathrm{C} 2-\mathrm{C} 3-\mathrm{C} 4-\mathrm{C} 5$ & $46.1(6)$ & $\mathrm{C} 12-\mathrm{C} 13-\mathrm{C} 14-\mathrm{C} 15$ & $-176.4(3)$ \\
\hline $\mathrm{C} 2-\mathrm{C} 3-\mathrm{C} 44^{\prime}-\mathrm{C} 5$ & $-40.0(10)$ & $\mathrm{C} 13-\mathrm{C} 14-\mathrm{C} 15-\mathrm{C} 16$ & $-173.8(3)$ \\
\hline $\mathrm{C} 3-\mathrm{C} 4{ }^{\prime}-\mathrm{C} 5-\mathrm{C} 6$ & $43.6(9)$ & $\mathrm{C} 14-\mathrm{C} 15-\mathrm{C} 16-\mathrm{C} 21$ & $-174.6(3)$ \\
\hline $\mathrm{O} 2-\mathrm{C} 4-\mathrm{C} 5-\mathrm{C} 6$ & $-174.4(5)$ & $\mathrm{C} 14-\mathrm{C} 15-\mathrm{C} 16-\mathrm{C} 17$ & $7.6(5)$ \\
\hline
\end{tabular}




$\mathrm{C} 3-\mathrm{C} 4-\mathrm{C} 5-\mathrm{C} 6$
$\mathrm{C} 7-\mathrm{C} 1-\mathrm{C} 6-\mathrm{O} 3$
$\mathrm{C} 2-\mathrm{C} 1-\mathrm{C} 6-\mathrm{O} 3$
$\mathrm{C} 7-\mathrm{C} 1-\mathrm{C} 6-\mathrm{C} 5$
$\mathrm{C} 2-\mathrm{C} 1-\mathrm{C} 6-\mathrm{C} 5$
$\mathrm{C} 4-\mathrm{C} 5-\mathrm{C} 6-\mathrm{O} 3$
$\mathrm{C} 4-\mathrm{C} 5-\mathrm{C} 6-\mathrm{O} 3$
$\mathrm{C} 4-\mathrm{C} 5-\mathrm{C} 6-\mathrm{C} 1$
$\mathrm{C} 4-\mathrm{C} 5-\mathrm{C} 6-\mathrm{C} 1$
$\mathrm{C} 6-\mathrm{C} 1-\mathrm{C} 7-\mathrm{O} 4$
$\mathrm{C} 2-\mathrm{C} 1-\mathrm{C} 7-\mathrm{O} 4$

$-39.9(6)$

$-0.4(4)$

$-178.6(3)$

$-179.6(3)$

$2.2(5)$

$155.7(5)$

$-163.2(4)$

$-25.0(6)$

$16.1(5)$

$-1.1(4)$

$177.0(3)$
$\mathrm{C} 21-\mathrm{C} 16-\mathrm{C} 17-\mathrm{C} 18$
$\mathrm{C} 15-\mathrm{C} 16-\mathrm{C} 17-\mathrm{C} 18$
$\mathrm{C} 16-\mathrm{C} 17-\mathrm{C} 18-\mathrm{C} 19$
$\mathrm{C} 17-\mathrm{C} 18-\mathrm{C} 19-\mathrm{O} 5$
$\mathrm{C} 17-\mathrm{C} 18-\mathrm{C} 19-\mathrm{C} 20$
$\mathrm{O} 5-\mathrm{C} 19-\mathrm{C} 20-\mathrm{C} 21$
$\mathrm{C} 18-\mathrm{C} 19-\mathrm{C} 20-\mathrm{C} 21$
$\mathrm{C} 19-\mathrm{C} 20-\mathrm{C} 21-\mathrm{C} 16$
$\mathrm{C} 17-\mathrm{C} 16-\mathrm{C} 21-\mathrm{C} 20$
$\mathrm{C} 15-\mathrm{C} 16-\mathrm{C} 21-\mathrm{C} 20$

$0.6(4)$

$178.4(3)$

$-0.4(5)$

$-179.9(3)$

$-0.2(4)$

$-179.6(3)$

$0.7(5)$

$-0.5(5)$

$-0.1(4)$

$-178.1(3)$

Hydrogen-bond geometry $\left(A,{ }^{o}\right)$

\begin{tabular}{lllll}
\hline$D-\mathrm{H} \cdots A$ & $D-\mathrm{H}$ & $\mathrm{H} \cdots A$ & $D \cdots A$ & $D-\mathrm{H} \cdots A$ \\
\hline $\mathrm{O} 2-\mathrm{H} 2 A \cdots \mathrm{O} 5^{\mathrm{i}}$ & 0.83 & 2.18 & $3.004(7)$ & 174 \\
$\mathrm{O} 2{ }^{\prime}-\mathrm{H} 2 B \cdots 5^{\mathrm{i}}$ & 0.82 & 1.86 & $2.565(16)$ & 143 \\
$\mathrm{O} 3-\mathrm{H} 3 O \cdots \mathrm{O} 4$ & 0.86 & 1.64 & $2.440(3)$ & 153 \\
$\mathrm{O} 5-\mathrm{H} 5 O \cdots \mathrm{O} 1^{i i}$ & 0.83 & 1.86 & $2.687(3)$ & 172
\end{tabular}

Symmetry codes: (i) $x+1, y-1, z+1$; (ii) $-x-1,-y+1,-z+1$. 